\title{
A MODEL-BASED STUDY OF TRANSMIT-RECEIVE LONGITUDINAL ARRAYS FOR INSPECTION OF SUBSURFACE DEFECTS
}

\author{
Ehsan Mohseni ${ }^{1}$, Charles Macleod, Yashar Javadi, \\ Randika Vithanage, Zhen Qiu, David Lines, Euan \\ Foster, Peter Lukacs, Momchil Vasilev, Rastislav \\ Zimermann, Gareth Pierce, Anthony Gachagan \\ Center for Ultrasonic Engineering \\ Department of Electronics and Electrical Engineering, \\ University of Strathclyde \\ Glasgow, UK
}

\begin{abstract}
Dual matrix transmit-receive longitudinal arrays have been shown to have an improved signal to noise ratio in near field zone which makes them the most suitable array configuration for inspection of near-surface defects. This study aims to compare the performance of different configurations for transmit-receive longitudinal matrix arrays. For this purpose, 4 matrix configurations of $2 \times 32,4 \times 16,4 \times 32$, and $8 \times 16$ elements are investigated using CIVA modeling. The array operating frequencies investigated are either $5 \mathrm{MHz}$ or $10 \mathrm{MHz}$. The effect of different natural focal depths, arrays' separation distances, dynamic electronic depth focusing, and electronic beam skewing for these TRL arrays are considered in models prepared in CIVA. The inspection of a series of flat bottom holes extended to a few millimeters under the surface using the selected TRL configurations is also investigated in the study. It is found that the focusing for near-surface areas is more efficient using the $4 \times 16$ and $8 \times 16$ elements configurations as compared to the others and the signal amplitudes of the defects located deeper in the target material are almost independent of the configuration.
\end{abstract}

Keywords: Transmit-receive longitudinal (TRL) matrix arrays, model-based ultrasonic studies, Non-destructive testing/evaluation, Matrix ultrasonic phased arrays

\section{NOMENCLATURE \& ABBREVIATIONS}

$\alpha \quad$ Arrays' relative separation angle

TRL Transmit-receive longitudinal

TRS Transmit-receive shear

NF Natural beam focus

$\mathrm{EF} \quad$ Electronic focus

FBH Flat-bottomed hole

SD Separation distance

\section{INTRODUCTION}

Dual element longitudinal transmit-receive ultrasonic transducers were frequently used in the past to detect corrosion and perform wall thickness measurements in different industrial sectors $[1,2]$. These transducers were widely accepted by the non-destructive testing community since the transmit-receive configuration minimizes the effect of the dead zone, resulting in an enhanced signal to noise ratio for near-surface inspections [3]. Inspired by the same design, the idea was extended to ultrasonic arrays including linear and matrix arrays [4]. These configurations, namely transmit-receive longitudinal (TRL) /shear (TRS) arrays, were soon adopted by the welding and joining community because of their increased sensitivity in noisy materials, such as austenitic stainless steels, relative to structural steels $[3,5,6]$. Moreover, as another advantage of TRL/TRS arrays, the ability to steer the beam in two-directions can be named [5]. However, in weld applications the inspections are majorly done by high angle beams and the probe's distance from the weld zone is reasonably larger than the near field zone associated with each array [6]. Hence, the previous studies fall short when it comes to the performance of such arrays for detection of near-surface defects where the defects are in the range of near field of the array and generating focal laws to focus the beam could affect the inspections' outcome [7]. The study is carried out in the framework of a larger project concerning the in-process inspection of Wire + Arc additive manufactured components $[7,8]$. The main strategy for such inspections is based on the contact ultrasound inspection using hightemperature wheel probes [9]. The probe's concept is comprised of a high-temperature outer shell made out of rubber, a hightemperature liquid fill with acoustically acceptable properties, and a pair of matrix arrays configured in TRL mode. The type of arrays, their configuration, and placement inside the wheel are of high importance since the depth covered by the formed beam using these arrays can be significantly affected by these setting. Therefore, the present study investigates the sensitivity of TRL arrays in the detection of defects that lie sub-surface (i.e. up to $40 \mathrm{~mm}$ deep under the surface). For this purpose, CIVA is used to model different configurations of TRL matrix arrays and their performance in detection of near-surface defects. CIVA has shown good flexibility and strength for array simulations in the past $[10,11]$, and therefore; employed here to study the effect of geometrical parameters of TRL configurations on their performance through the quality of the signal. Here, as

\footnotetext{
${ }^{1}$ Contact author: ehsan.mohseni@strath.ac.uk
} 
demonstrated in Figure 1, the term performance stands for the ability to focus the probe's beam in close proximity to the material front surface, beam steering in primary inspection plane (i.e. the plane aligned with the length of the array and mirroring the transmission array to the reception one), and beam skewing in the secondary inspection plane (i.e. the plane cutting the arrays' length in half). To address these, four TRL probes, all having a zero-degree stand-off from the material surface, are considered in the study.

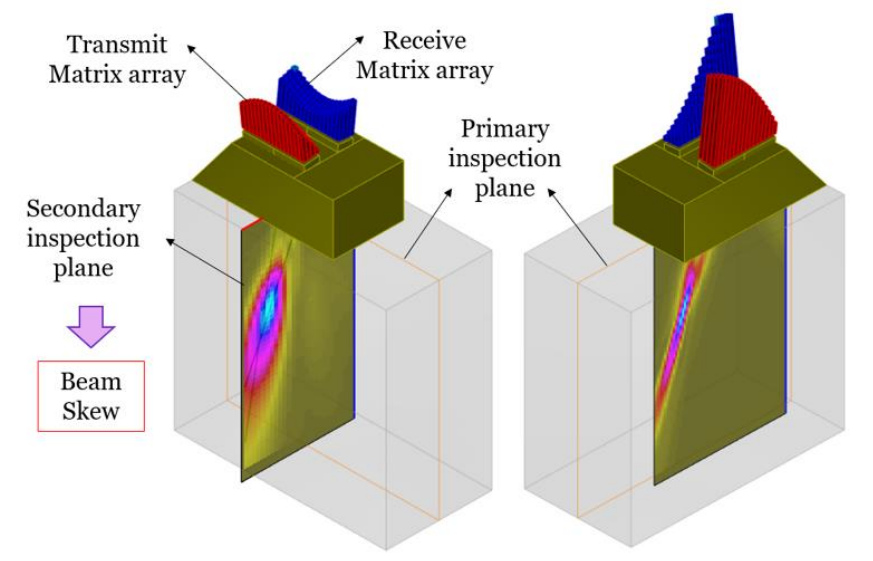

FIGURE 1: BEAM STEERING AND SKEWING IN THE PRIMARY AND SECONDARY INSPECTION PLANES, RESPECTIVELY

\section{MATERIALS AND METHODS}

A number of matrix arrays on stand-off wedges, made out of Rexolite, with dimensions of $40 \mathrm{~mm} \times 20 \mathrm{~mm} \times 15 \mathrm{~mm}$ are modeled in CIVA. As depicted in Figure 2(a), the two arrays mounted on the wedge have a separation angle and distance, which can affect the quality of the beamforming. Four different matrix arrays with layouts of $2 \times 32,4 \times 16,4 \times 32$, and $8 \times 16$ elements are designed where the first two operate at $5 \mathrm{MHz}$ and have 64 channels in transmitting and receiving, as can be seen in Figure 2(b). The $5 \mathrm{MHz}$ arrays have square elements with size of $0.4 \mathrm{~mm}$ with a separation of $0.1 \mathrm{~mm}$ in both matrix dimensions. The second pair of matrix arrays have 128 channels of transmission and reception with two layouts of $4 \times 32$ and $8 \times 16$ elements, and they operate at a frequency of $10 \mathrm{MHz}$. The square elements of these arrays are $0.2 \mathrm{~mm}$ long with a separation of 0.1 $\mathrm{mm}$ in both directions of the matrix. It should be noted that the element dimensions are selected to approximately have an element pitch equal to the half of wavelength in titanium at the operating frequency. Theoretically, this consideration leads to 90 degrees of separation between the grating lobes and the main beam if the array is directly coupled to a titanium sample [12, 13]. However, these element sizes are not optimal anymore when a pair of the arrays are used in the TRL configuration and they are positioned on a stand-off wedge. The effect of pitch on the separation angle of grating lobes is presented in the final section as a 2-D matrix array is placed in contact with a Rexolite wedge. Moreover, the section investigates how large elements can grow in the second dimension of a 2-D matrix array (i.e. arrays' width) without impairing the functionality of the array in beam skew ability [14]. Therefore, as illustrated in Figure 3, the beam generated by an $8 \times 16$ matrix array $(10 \mathrm{MHz})$ in a Rexolite medium is analyzed in the primary and secondary inspection planes, to plot the main beam and the side lobes formed in each plane. The elements with the initial size of $0.3 \mathrm{~mm}$ are resized along the second dimension of the array, containing 8 elements, to reach the size of $0.9 \mathrm{~mm}$ in 3 steps. Having larger elements is desirable for increasing the beam energy which could be beneficial in inspection of/through attenuative materials. This is particularly of interest in the current study since the arrays are being configured to perform within a wheel probe and inspect the target material (i.e. titanium) through a high-temperature rubber insulator (i.e. attenuative layer).

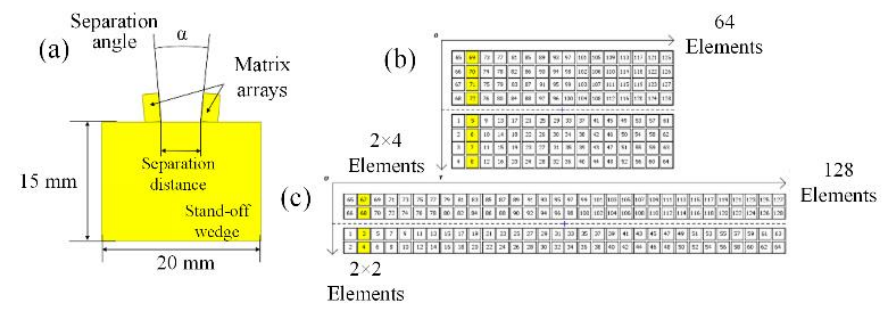

FIGURE 2: (a) ASSEMBLY OF THE STAND-OFF WEDGE AND MATRIX ARRAYS, AND LAYOUT FOR 64 CHANNELS TRANSMIT AND RECEIVE MATRIX ARRAYS OF (b) $2 \times 32$, AND (c) $4 \times 16$.

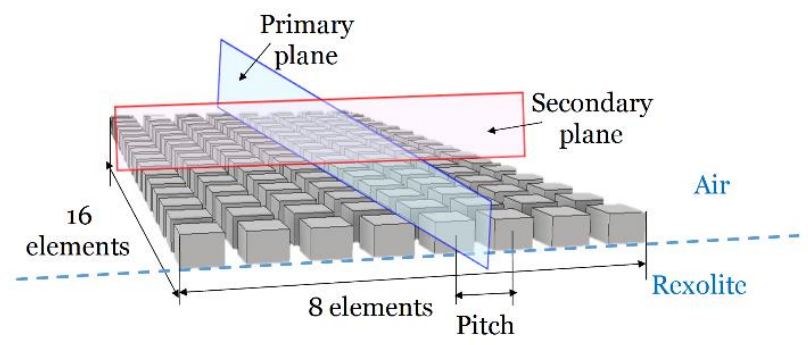

FIGURE 3: A $8 \times 16$ MATRIX ARRAY POSITIONED ON A REXOLITE MEDIUM WITH THE PRIMARY PLANE CUTTING THE ARRAY'S GEOMETRY IN HALF ACROSS ITS WIDTH AND THE SECONDARY PLANE CUTTING THE GEOMETRY OF THE ARRAY IN HALF ACROSS ITS LENGTH

A titanium block with dimensions of $100 \times 60 \times 30 \mathrm{~mm}$ is placed beneath the wedge and arrays assembly. In the first stage of simulations, the block does not contain any defects since the aim is to conduct a study on the beamforming ability. To this end, the arrays' relative angle $(\alpha)$ is varied in a manner to have a natural beam focus (NF) at $5 \mathrm{~mm}, 10 \mathrm{~mm}$, and $20 \mathrm{~mm}$ under the interface of the wedge and the material while the separation 
distance (SD) is fixed at $5 \mathrm{~mm}$. Also, in order to assess the effect of SD variations on the beam amplitude, $3 \mathrm{SD}$ values of $5 \mathrm{~mm}$, $10 \mathrm{~mm}$, and $15 \mathrm{~mm}$ are used to separate the arrays while the NF is maintained to be at the depth of $10 \mathrm{~mm}$. For the aforementioned combinations, the focal laws are generated to change the electronic focus (EF) at different depths varying from $3 \mathrm{~mm}$ to $30 \mathrm{~mm}$.

In the second stage of the study, $\alpha$ is set to have the natural focus fixed at the depth of $10 \mathrm{~mm}$ in a titanium block containing flat bottom holes (FBH) for each of the matrix array configurations. These holes extend from the lower surface of the block to different depths under the wedge/block interface. The distance from the wedge/block interface to the tip of the hole (ligament) is changed from $3 \mathrm{~mm}$ to $39 \mathrm{~mm}$ with increments of $3 \mathrm{~mm}$. The holes' centerline relative to the wedge center point is changed to compare the amplitude of FBH indications for different matrix configurations, and skew angles. These different inspection setups are named and listed as follows:

1) In-plane centered (Mode 1): The FBH's centerline is centered with the wedge to get an insight into the effectiveness of multi-depth focusing for different matrix configurations. The wedge is placed on each hole to carry out the inspection as demonstrated in Figure 4(a).

2) Out of plane (Mode 2): FBHs are offset by $5 \mathrm{~mm}$ from the primary inspection plane. This setup, as depicted in Figure 4(b), is meant for studying the ability of beam skewing for different TRL configurations.

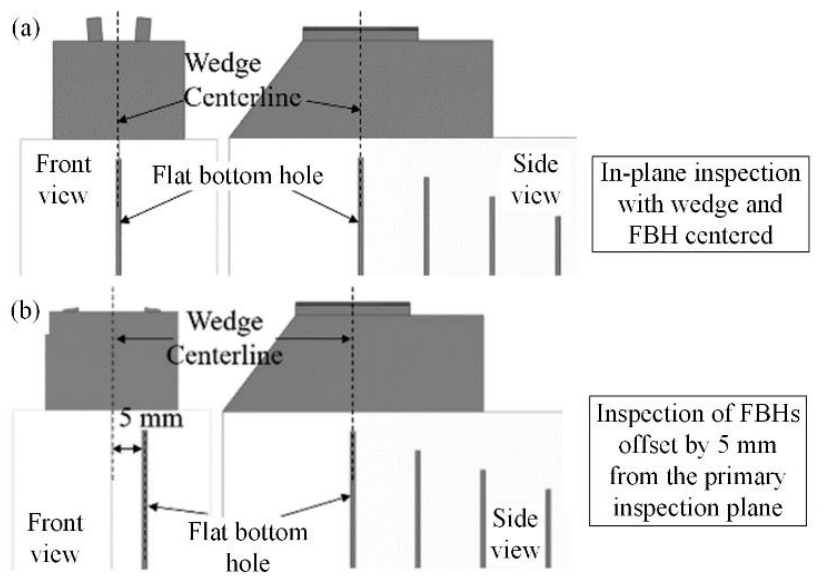

FIGURE 4: POSITIONING OF THE WEDGE WITH RESPECT TO THE FLAT BOTTOM HOLES IN THE SETUP PREPARED TO STUDY (a) DYNAMIC DEPTH FOCUSING, AND (b) BEAM SKEWING.

\section{RESULTS AND DISCUSSION}

Figures 5(a) and 5(b) plot the results of the study of beam focusing for two arrays of $4 \times 16$ and $2 \times 32$, respectively. Here, the maximum amplitude of the beam is plotted versus the nominal depth of EF. It should be noted that all the amplitude levels presented in Figure 5 are normalized with respect to the maximum beam amplitude of a $2 \times 32$ TRL when the NF is at 10 $\mathrm{mm}, \mathrm{EF}$ is at $3 \mathrm{~mm}$ and SD is set to be $10 \mathrm{~mm}$. The results confirm the higher amplitude level of the focal spot for the formed beam of a $4 \times 16$ TRL. One would expect the higher amplitude of a $4 \times 16$ TRL because of the 4 elements that are involved in beam skew in the secondary plane. In addition, when the beam is focused at a certain depth, the acoustic field of a $4 \times 16$ TRL forms a pyramid to which the matrix array is the base. This pyramidal beam is narrower and more concentrated for a $4 \times 16$ matrix, owing to the shape of its base being closer to a square, giving rise to a higher beam amplitude. The amplitude of this configuration is higher by approximately $4 \mathrm{~dB}$ regardless of the depth of focus. The trend of the amplitude reduction for a $4 \times 16$ TRL, as the focal depth increases, closely follows the one obtained for a $2 \times 32$ TRL. It is also notable that the discrepancy between the amplitude of different NF values is very small for both $2 \times 32$ and $4 \times 16$ configurations meaning that the signal amplitude is insensitive to the small variations of $\alpha$.

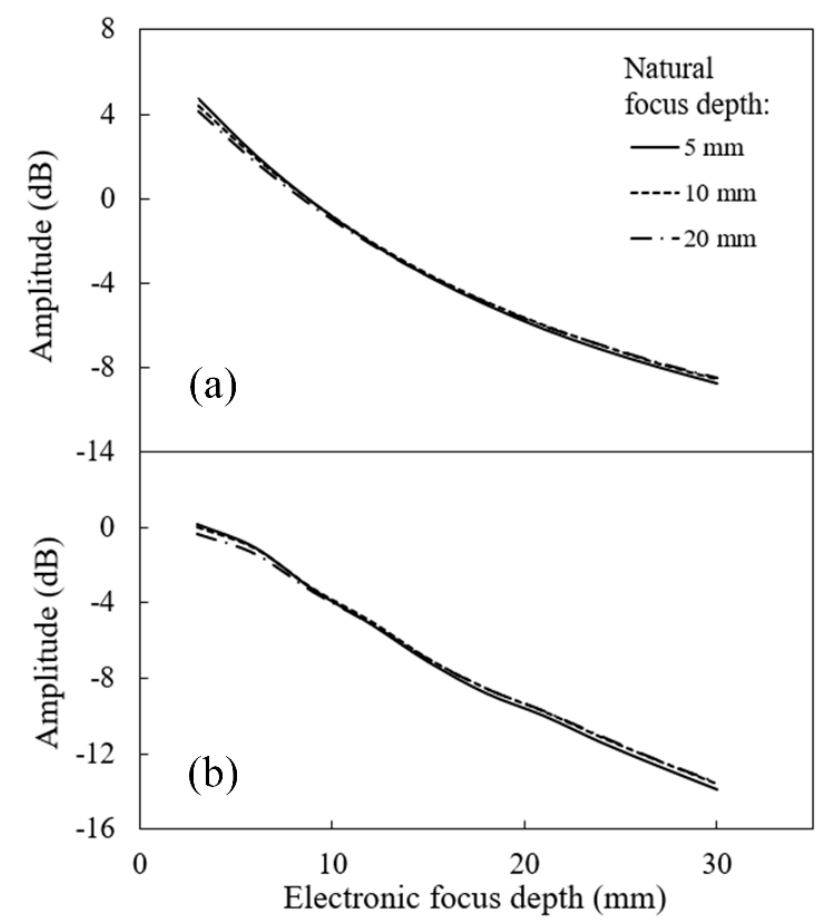

FIGURE 5: VARIATIONS OF THE MAXIMUM AMPLITUDE OF THE FOCAL SPOT VERSUS THE NOMINAL FOCUSING DEPTH AS THE NATURAL FOCAL DEPTH IS CHANGED IN 3 STEPS FOR (a) A 4×16, AND (b) A $2 \times 32$ MATRIX ARRAY

Although the amplitude results presented in Figure 5 are plotted versus the electronic focus depth, the actual position of the focal spot doesn't always match the EF. To clarify this, in Figure 6, EF is changed from $3 \mathrm{~mm}$ to the depth of $27 \mathrm{~mm}$ in steps of $6 \mathrm{~mm}$ for both TRL configurations. The focal spot for each of these computed beams is plotted for $12 \mathrm{~dB}$ drop in amplitude where the $0 \mathrm{~dB}$ stands for the maximum amplitude. The color bar is the same for all these plots presented in the 
figure however, the amplitude values are not normalized. This means the results obtained for a certain $\mathrm{EF}$ is not comparable to the others in terms of the signal amplitude. Figure 6 clearly shows that the depth of almost all EF values is underestimated by the actual position of the beam's maximum. This difference between the preset EF and the actual position of the focal spot obtained by a $4 \times 16$ TRL is twice the one simulated for a $2 \times 32$ TRL. The discrepancy can be explained by the stronger steerability of the $2 \times 32 \mathrm{TRL}$, in the primary inspection plane, since the number of elements stacked in the dimension of array parallel to the primary inspection plane is more (i.e. 32 elements). Moreover, the $\alpha$ angle is larger for a $4 \times 16$ TRL when the same NF is set for both configurations. Taking into account the larger $\alpha$ angle for a $4 \times 16$ TRL, the beam should be skewed more to reach a certain EF depth which can cause the beam to approach the physical limits of the array faster, and hence; resulting in a reduction in the depth of dynamic focus. The figure also suggests that the shape of focal spot is affected by the matrix layout as the amplitude range of $0 \mathrm{~dB}$ to $-12 \mathrm{~dB}$ plotted for the focal spot of a $4 \times 16$ TRL forms a vertically expanded ellipse whereas the elliptical form of the spot is aligned horizontally for $2 \times 32$ TRL. This observation can be explained by the ratio of the number of elements across the length to width $(\mathrm{L} / \mathrm{W})$ of a $2 \times 32$ TRL.

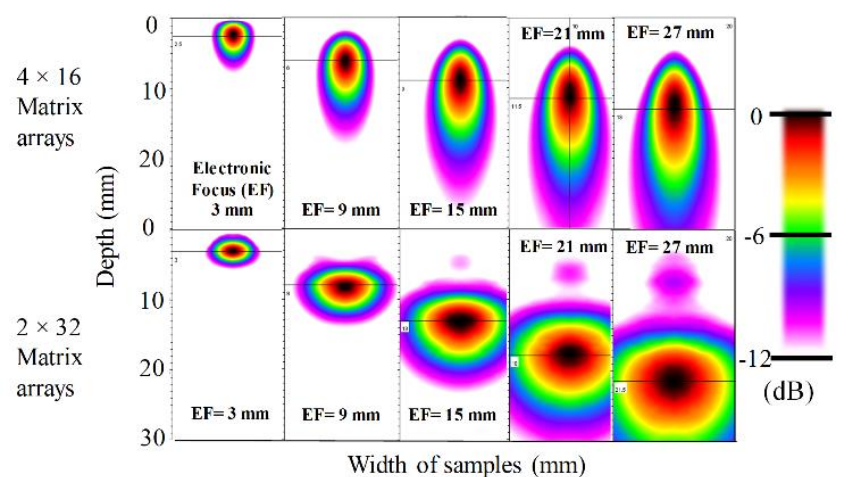

FIGURE 6: CHANGES IN THE SHAPE OF FOCAL SPOT AS THE ELECTRONIC FOCUS VARIES FROM DEPTH OF 3 MM TO 27 MM IN 5 STEPS FOR (a) A $4 \times 16$, AND (b) A $2 \times 32$ MATRIX ARRAY.

The mismatch in the position of EF and the focal spot is also demonstrated in Figure 7 graphs. The figure shows the profile of beam energy along the central vertical line of each focal spots plotted in Figure 6. As an instance, the peak of the curve plotted for a $4 \times 16 \mathrm{TRL}$, when the EF is $27 \mathrm{~mm}$, occurs at the depth of $10 \mathrm{~mm}$. On the other hand, the peak is located at a depth of 20 $\mathrm{mm}$ for the same EF for a $2 \times 32 \mathrm{TRL}$. The beam profile for a $4 \times 16$ TRL configuration has a smoother transition from its peak to lower amplitudes.

Figures 8(a) and 8(b) plot the maximum amplitude of the beam (i.e. focal spot) versus the actual focal depth for different arrays' separation distances for two TRL configurations of $4 \times 16$ and $2 \times 32$. The same normalization scheme used in Figure 4 is deployed here. As expected, the maximum beam amplitude of a $4 \times 16$ is always higher at a selected SD.

The maximum amplitude of the focal spot changes significantly as the SD is varied from $5 \mathrm{~mm}$ to $15 \mathrm{~mm}$ at shallow focal depths, and this change is more pronounced for a $4 \times 16 \mathrm{TRL}$ indicating the higher sensitivity of a $4 \times 16$ TRL to SD. Moreover, the actual position of the focal spot is also affected by SD variations when one uses a $4 \times 16$ TRL. As can be seen in Figure 8(a), The difference between the EF of $27 \mathrm{~mm}$ and the actual depth of focus grows larger when the SD is lowered to $5 \mathrm{~mm}$. Despite having higher focal amplitudes at lower SD values for a $4 \times 16$ TRL, the allowable dynamic focusing depth shrinks. On the other hand, changing the SD value has a minimal effect on the actual focus of a $2 \times 32$ array however, it still influences the maximum amplitude.

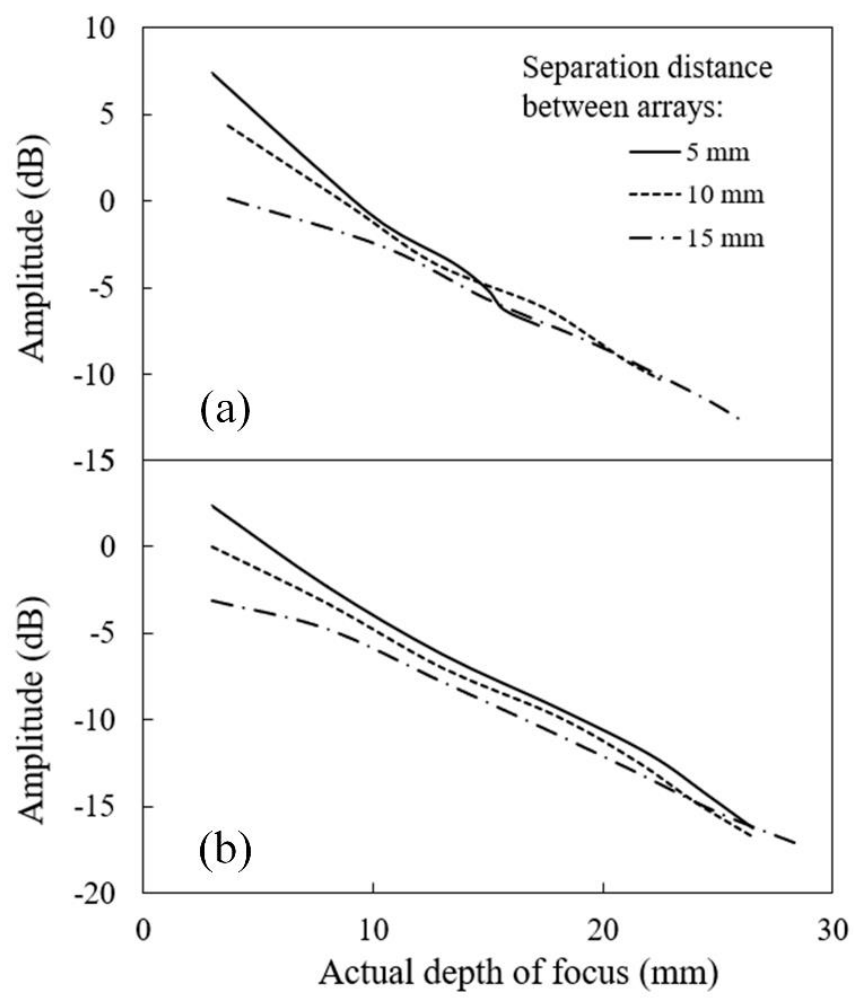

FIGURE 7: VARIATIONS OF THE MAXIMUM AMPLITUDE OF THE FOCAL SPOT VERSUS THE FOCUSING DEPTH AS THE ELECTRONIC FOCUS CHANGES FROM 3 MM TO 27 MM IN STEPS OF 3 MM FOR (a) A 4×16, AND (b) A $2 \times 32$ MATRIX ARRAYS 


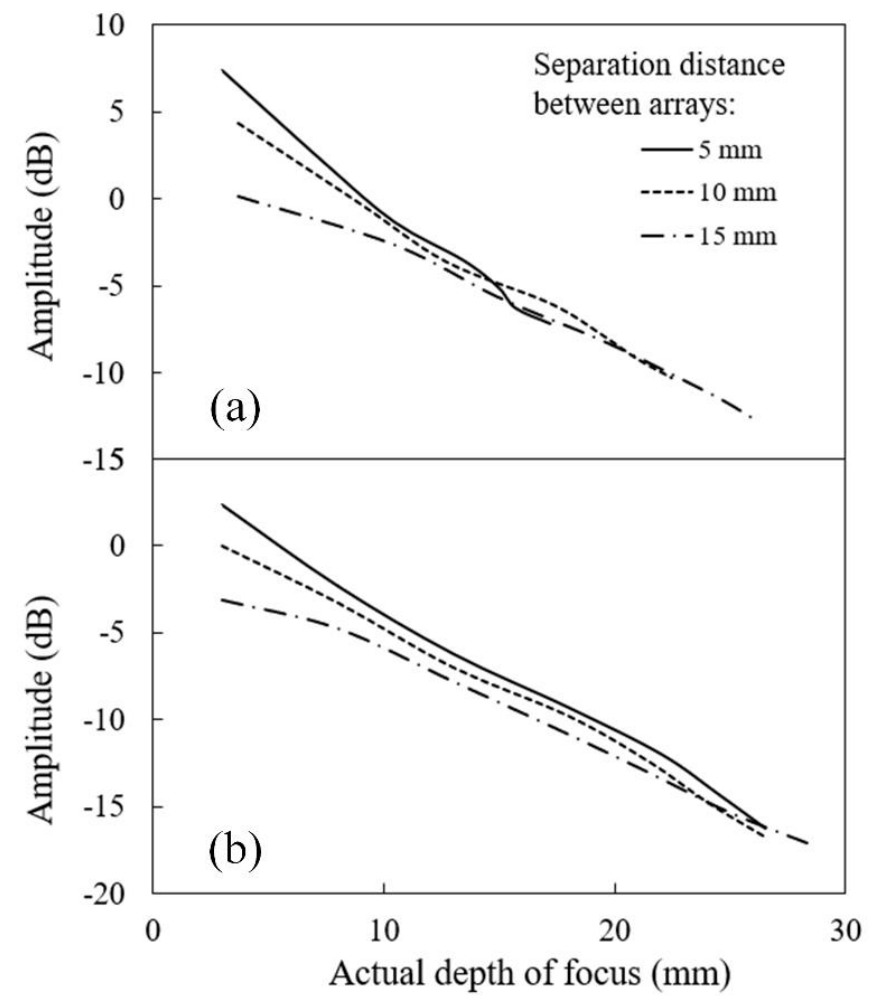

FIGURE 8: VARIATIONS OF THE MAXIMUM AMPLITUDE OF THE FOCAL SPOT VERSUS THE ACTUAL FOCUSING DEPTH AS THE SEPARATION DISTANCE BETWEEN THE ARRAYS CHANGES FROM 5 MM TO 15 MM IN 3 STEPS. RESULTS ARE PROVIDED FOR (a) A $2 \times 32$ MATRIX ARRAY, AND (b) A 4×16 MATRIX ARRAY

Figure 9 plots the results concerning the second stage of this study for inspection of FBHs with different ligaments using the two TRL configurations of $2 \times 32$ and $4 \times 16$ operating at a frequency of $5 \mathrm{MHz}$. The figure compares the In-pane inspections with the out of plane inspections for the two configurations. The results suggest that the signal amplitudes are always higher when using a $4 \times 16$ TRL configurations however, this superiority diminishes as deeper FBHs are scanned. Accordingly, it is safe to state that the configuration of matrix TRLs has a significant impact on the signal of near-surface defects. Looking at the results of mode 2, it is evident that for FBHs having a ligament of smaller than $9 \mathrm{~mm}$, the signal amplitude is very low as compared to the amplitudes obtained for the same FBHs in mode 1 inspections. This is mainly due to the limited skew ability for very close to surface FBHs. Therefore, when the inspections are carried out in mode 2 where the beam is needed to be skewed, defects should be sufficiently far from the transducer in order to gain a better signal to noise $(\mathrm{S} / \mathrm{N})$ ratio. For TRL arrays of $2 \times 64$ channels in use, this threshold distance appears to be equal to $9 \mathrm{~mm}$. The amplitude difference between the 2 scan modes reduces as the ligament increases to $39 \mathrm{~mm}$ where the beam only needs to be skewed a few degrees in mode 2.

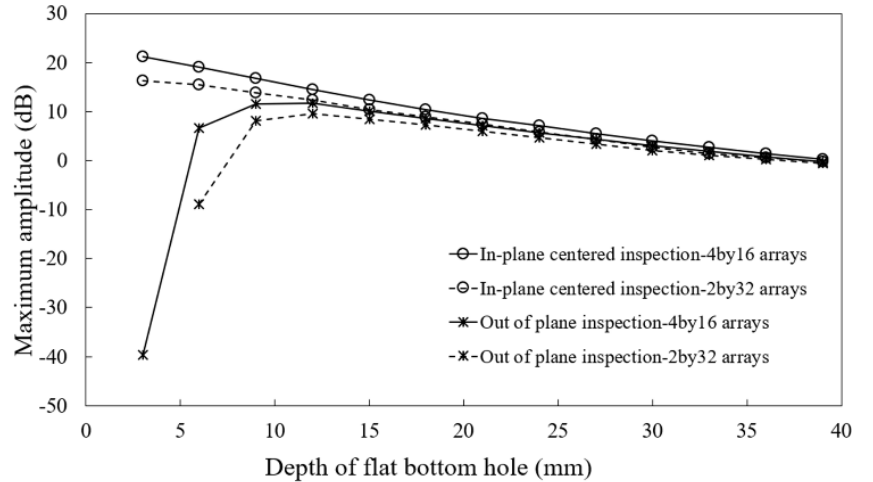

FIGURE 9: AMPLITUDE OF FLAT BOTTOM HOLES LOCATED AT DIFFERENT DEPTHS UNDER THE WEDGE/BLOCK INTERFACE. IN-PLANE AND OUT OF PLANE INSPECTIONS USING TWO TRL MATRIX ARRAYS OF $2 \times 32$ AND $4 \times 16$

Figures 10(a) and 10(b) present the plotted data for modes 1 and 2, respectively when the SD is also changing from $5 \mathrm{~mm}$ to $15 \mathrm{~mm}$ in 3 steps. Both of these figures show that signal amplitude is always higher for a $4 \times 16$ TRL array, and the amplitude discrepancy between the two configurations decreases as the ligament increases. Lowering the SD from $15 \mathrm{~mm}$ to $5 \mathrm{~mm}$ would increase the signal amplitude by $15 \mathrm{~dB}$ for inspecting a 3 $\mathrm{mm}$ FBH in mode 1, as suggested by Figure 10(a). This amplitude difference is very significant and is the evidence for the high sensitivity of the inspection response to SD. The results of mode 2 inspections plotted in Figure 10(b) are more complicated. It is mainly implying that putting the TRL arrays closer to each other makes it more difficult to skew the beam out of the inspection plane, particularly for very close to surface FBHs. As it can be seen the signal amplitude of FBHs with ligaments of smaller than $12 \mathrm{~mm}$ is very low, but the signal amplitude for these FBHs improves as the SD is increased to 15 $\mathrm{mm}$. This improvement is accompanied with lowering the amplitudes recorded for deeper FBHs. Considering the two figures at once, a better signal for close to surface defects is achieved by lower SD however, this would limit the beam skew angle significantly as well leading to a very low signal amplitude of defects close to the surface in mode 2. Thus, the array's separation should be optimized to keep the skew ability within an acceptable range while the signal amplitude of the in-plane inspections is not sacrificed considerably. 


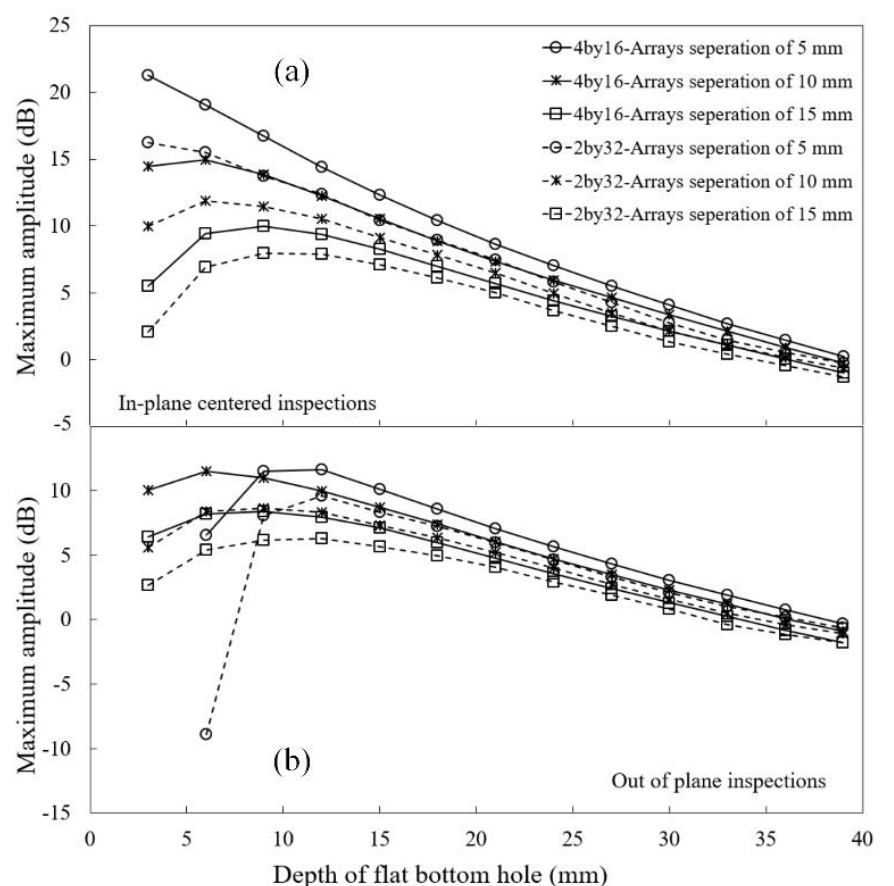

FIGURE 10: AMPLITUDE OF FLAT BOTTOM HOLES LOCATED AT DIFFERENT DEPTHS UNDER THE WEDGE/BLOCK INTERFACE AS THE SEPARATION DISTANCE BETWEEN THE ARRAYS CHANGES FROM 5 MM TO 15 MM IN 3 STEPS. INSPECTIONS ARE PERFORMED USING TWO TRL MATRIX ARRAYS OF $2 \times 32$ AND $4 \times 16$ IN MODES (a) IN-PLANE AND (b) OUT OF PLANE

The results of modes 1 and 2 inspections using $2 \times 128$ channels TRLs operating at $10 \mathrm{MHz}$ are plotted in Figure 11. One can spot the lower amplitude levels for these as compared to the graphs presented in Figure 9 for $5 \mathrm{MHz}$ tests with $2 \times 64$ channels. The lower amplitude in Figure 11 is clearly originated from the smaller surface area of $10 \mathrm{MHz}$ arrays leading to lower beam energy and hence, signal amplitudes. Moreover, Figure 11 demonstrates that, regardless of inspection mode, the signal amplitude obtained for the FBHs is always higher using an $8 \times 16$ array. This is in agreement with the case of $2 \times 64$ channels operating at $5 \mathrm{MHz}$ where the signal amplitude for the arrays with a layout closer to a square (i.e. $4 \times 16$ ) is higher. On another feature of this graph, the signal amplitude for mode 2 inspections is very low when the FBHs are extended very close to the inspection surface. These amplitudes start to increase until they gradually reach a peak which occurs at the depth of $12 \mathrm{~mm}$. This threshold depth was also present in Figure 9 for $5 \mathrm{MHz}$ inspections however, in that case, the threshold was closer to the depth of $9 \mathrm{~mm}$. The comparison between these two figures and the threshold depths imply that the success rate to capture shallower defects falling out of the primary inspection plane is higher using $2 \times 128$ TRL arrays rather than $2 \times 64$ arrays since the increased number of elements enhances the beam skew abilities. Following the case investigated in Figure 9, it also can be observed in Figure 11 that the signal amplitudes recorded using different modes and configurations tend to converge as the FBH depth increases showing the TRL's high sensitivity to its configuration for inspection of close to surface defects.

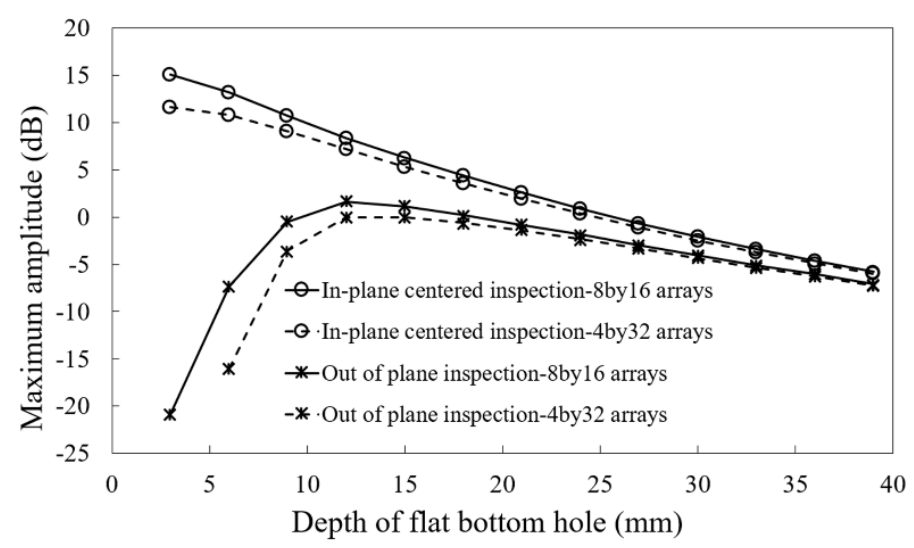

FIGURE 11: AMPLITUDE OF FLAT BOTTOM HOLES LOCATED AT DIFFERENT DEPTHS UNDER THE WEDGE/BLOCK INTERFACE. IN-PLANE AND OUT OF PLANE INSPECTIONS USING TWO TRL MATRIX ARRAYS OF $4 \times 32$ AND $8 \times 16$

Figures 12 (a) and (b) show the results of modes 1 and 2 inspections utilizing the 2 different TRL configurations at 10 $\mathrm{MHz}$ as the SD is changed from $5 \mathrm{~mm}$ to $15 \mathrm{~mm}$ in 3 steps. Again these results conform with ones presented in Figure 10, and they suggest a better inspection performance of a pair of arrays with dimensions closer to a square (i.e. $8 \times 16$ elements) over a $4 \times 32$ TRL configuration. According to Figure 12(a), similar to the $5 \mathrm{MHz}$ case presented in Figure 10 (a), the increased separation of arrays reduces the signal amplitudes for mode 1 inspections, especially for the FBHs extended closer to the inspection surface. The graphs confirm that the signal amplitude of shallow subsurface FBHs can be severely affected by the changes in the separation distance.

As depicted in Figure 12(b), the signal amplitudes of shallow FBHs inspected in mode 2 improve as the SD is increased. As stated earlier, placing the arrays further away from each other allows the beam to be skewed up to higher angles, and accordingly; increasing the signal amplitude of the FBHs located out of the primary inspection plane. However, the signal amplitude decreases by increasing the SD value for the FBHs located deeper than the threshold depth of $12 \mathrm{~mm}$ since these FBHs are captured by skewing the beam only by a few degrees. Noteworthy that the signal amplitudes converge for all inspection modes and configurations as deeper FBHs are inspected.

The effect of matrix array's layout on the ability of beam skew is briefly studied in the previous sections however, the presented results mainly discuss the maximum beam amplitude and the FBHs' signal amplitude. These two are reliable measures to assess the performance of a configuration with, however they are not sufficient since the noise is not incorporated in them. A part of the noise attributes to the structural features which can bring about a slight difference between the results of different array configurations studied here. However, there would be a 
significant contribution from the grating lobes to the faulty signals (e.g. ghost signals) when the beam is being skewed. Hence, it is of high importance to extend the scope of the study further in order to investigate the limitations in the beam skew dictated by the grating lobes when the 2-D arrays are used for modes 1 and 2 inspections. A model of an $8 \times 16$ 2-D array was built in MATLAB (R2018b, MathWorks) using Huygens-Fresnel principle, at the test frequency of $10 \mathrm{MHz}$ [15]. The focus was set to be $50 \mathrm{~mm}$ in Rexolite medium, within the transducer near field. Consequently, the beam main lobe and grating lobes are plotted in the primary (blue-bordered image) and secondary (redbordered image) planes in Figure 13.

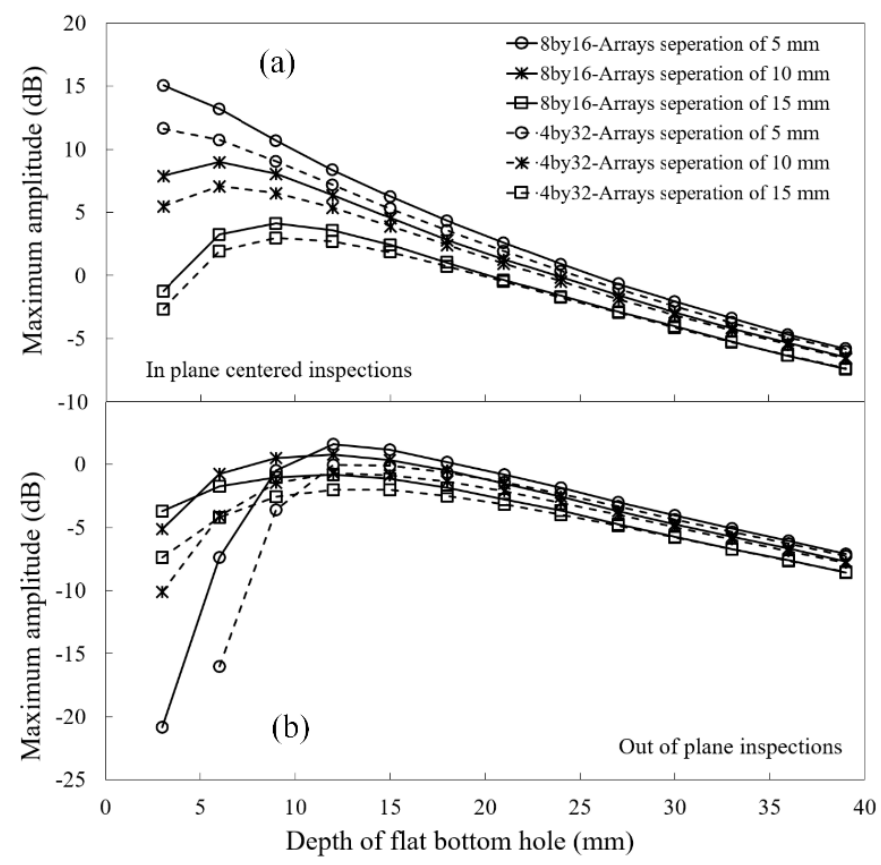

FIGURE 12: AMPLITUDE OF FLAT BOTTOM HOLES LOCATED AT DIFFERENT DEPTHS UNDER THE WEDGE/BLOCK INTERFACE AS THE SEPARATION DISTANCE BETWEEN THE ARRAYS CHANGES FROM 5 MM TO 15 MM IN 3 STEPS. INSPECTIONS ARE PERFORMED USING TWO TRL MATRIX ARRAYS OF $4 \times 32$ AND $8 \times 16$ IN MODES (a) IN-PLANE AND (b) OUT OF PLANE

The Figures 13(a), 13(b) and 13(c) depict the results for element pitches of $0.3 \mathrm{~mm}, 0.6 \mathrm{~mm}$, and $0.9 \mathrm{~mm}$, along the width of the array (i.e. the secondary plane shown in Figure 3) containing 8 elements, respectively. The main beam is received at 0 degree and the other grating lobes are separated by different angles from the main beam. The images presented in the first row of this figure show that the change of the element pitch along the array width barely influences the number of grating lobes in the primary plane of inspection. However, the amplitude of the main beam and the grating lobes reduces in this plane as the pitch is increased to $0.9 \mathrm{~mm}$. The lower amplitude of the beam from the array with $0.9 \mathrm{~mm}$ pitch is due to the lower focus-ability of an array with larger elements. The images related to the secondary inspection plane are presented in the second row of Figure 13.
According to these images, the separation of the main beam and adjacent grating lobes decreases significantly as the pitch is increased to $0.9 \mathrm{~mm}$. The separation angle between the main beam and grating lobe decreases to 20 degrees meaning that the inspection beam skew angle in a Rexolite wedge becomes limited to less than 20 degrees in each direction. As the beam is skewed close to the 20 degrees limit, the signal gets affected by the reflections of the grating lobes. Therefore, it is essential to account for the effect of grating lobes when deciding about the element pitch. Increasing the beam energy comes at the price of tightening the steer/skew ability.

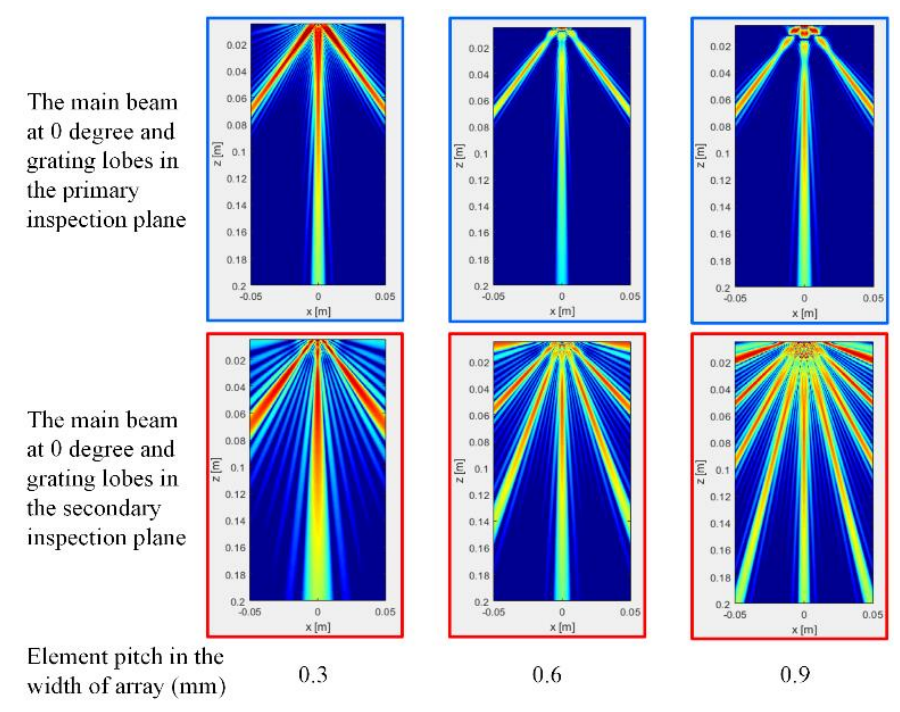

FIGURE 13: MAIN BEAM AND GRATING LOBES OF A $8 \times 16$ ARRAY AT 10 MHZ ARE PLOTTED IN THE PRIMARY AND SECONDARY INSPECTION PLANES OF A REXOLITE MEDIUM WHEN THE ELEMENT PITCH ALONG THE ARRAYS' WIDTH IS EQUAL TO (a) $0.3 \mathrm{MM}$, (b) $0.6 \mathrm{MM}$, AND (c) $0.9 \mathrm{MM}$

\section{CONCLUSIONS}

A number of simulations are carried out using CIVA in order to investigate the performance of different configurations of TRL matrix arrays operating at 2 frequencies of $5 \mathrm{MHz}$ and $10 \mathrm{MHz}$. According to the results of these simulations, the study can be concluded as follows:

- With a fixed number of channels, stacking elements in a way to have a matrix shape closest to a square would improve the beam skew ability. However, there is a trade-off between beam skew and beam steerability.

- The effect of small changes in the natural focus of TRL on the maximum amplitude of the focal spot, as the beam is focused at different depths, is trivial.

- The actual focusing depth is always lower than the nominal one introduced by focal laws. This difference is larger for matrix arrays with a shape closer to a square. 
- Increasing the separation distance between arrays lowers the signal amplitude of FBHs located in the central inspection plane, and the intensity of the focal spot for $5 \mathrm{MHz}$ and $10 \mathrm{MHz}$ inspections. This effect is much stronger for the FBHs extended closer to the inspection surface.

- The signal amplitude recorded by a pair of 64 channels matrix arrays is very poor for the FBHs located up to the depth of $9 \mathrm{~mm}$ off the center of inspection plane since the beam shall be skewed to very high angles, which is not physically achievable.

- The signal amplitude recorded by a pair of 128 channels matrix arrays is very low for the FBHs located up to the depth of $9 \mathrm{~mm}$ off the center of inspection plane, especially when the separation distance for the arrays is small.

- The dependency of the signal amplitude on the value of separation distance and the type of configuration decreases as one inspects deep FBHs at either $5 \mathrm{MHz}$ or $10 \mathrm{MHz}$.

- The effect of grating lobes shall be considered in beamforming to either skew or steer the beam. Larger elements allow to gain higher beam energies however, this would tighten the separation angle between the main beam and the grating lobes.

\section{ACKNOWLEDGMENT}

The research was carried out towards the objectives of Wire + Arc Additive Manufacturing (WAAM) projects, in collaboration with Welding Engineering and Laser Processing Centre of Cranfield University, which are all supported by EPSRC and Innovate UK as follows: (I) NEWAM (EP/R027218/1), (II) RoboWAAM (EP/P030165/1), (III) OAAM (113164), and (IV) AIMaReM (EP/N018427/1). The authors would like to acknowledge EPSRC, Innovate UK, and RCNDE for the support and funding of the projects.

\section{REFERENCES}

1. Lebowitz, C.A. and L.M. Brown, Ultrasonic measurement of pipe thickness, in Review of Progress in Quantitative Nondestructive Evaluation. 1993, Springer. p. 1987-1994.

2. Lozev, M., R. Smith, and B. Grimmett, Evaluation of methods for detecting and monitoring of corrosion damage in risers. 2005.

3. Juengert, A., et al. Advanced ultrasonic techniques for nondestructive testing of austenitic and dissimilar welds in nuclear facilities. in AIP Conference Proceedings. 2018. AIP Publishing LLC.

4. Nageswaran, C., C. Bird, and A. Whittle, Immersion transmit-receive longitudinal phased array probe for stainless steel. Insight-Non-Destructive Testing and Condition Monitoring, 2008. 50(12): p. 673-678.

5. Wang, R., et al., Research on phased array ultrasonic testing on CFETR vacuum vessel welding. Fusion Engineering and Design, 2019. 139: p. 124-127.

6. Kumar, S., M. Menaka, and B. Venkatraman, Simulation and experimental analysis of austenitic stainless steel weld joints using ultrasonic phased array. Measurement Science and Technology, 2019. 31(2): p. 024005.

7. Williams, S.W., et al., Wire+ arc additive manufacturing. Materials Science and Technology, 2016. 32(7): p. 641-647.

8. Martina, F., et al., Tandem metal inert gas process for high productivity wire arc additive manufacturing in stainless steel. Additive Manufacturing, 2019. 25: p. 545-550.

9. Mohseni, E., et al. Ultrasonic phased array inspection of wire plus arc additive manufactured (WAAM) titanium samples. in 58th Annual British Conference on Non-Destructive Testing. 2019.

10. Mahaut, S., et al., Recent advances and current trends of ultrasonic modelling in CIVA. Insight-NonDestructive Testing and Condition Monitoring, 2009. 51(2): p. 78-81.

11. Chaffaï-Gargouri, S., et al. Simulation and Data Processing for Ultrasonic Phased-Arrays Applications. in AIP Conference Proceedings. 2007. American Institute of Physics.

12. Steinberg, B.D., Principles of aperture and array system design: Including random and adaptive arrays. wi, 1976.

13. Wooh, S.-C. and Y. Shi, Optimum beam steering of linear phased arrays. Wave motion, 1999. 29(3): p. 245-265.

14. Wooh, S.-C. and Y. Shi, Influence of phased array element size on beam steering behavior. Ultrasonics, 1998. 36(6): p. 737-749.

15. Rasmussen, M.F., et al., 3-D imaging using rowcolumn-addressed arrays with integrated apodizationPart I: Apodization design and line element beamforming. IEEE transactions on ultrasonics, ferroelectrics, and frequency control, 2015. 62(5): p. 947-958. 